\title{
Dural metastasis of prostate adenocarcinoma with spontaneous subdural hematoma: an unusual mimicker of meningioma
}

Metástase dural solitária de adenocarcinoma de próstata mimetizando meningioma: disseminação incomum de uma neoplasia frequente Marianna CIONI', Mateos BOGONI', Carolina CHOMISKI', Laura MASCHKE', Marco Aurélio Soato RATTI', Bernardo Corrêa de Almeida TEIXEIRA

A 65-year-old male presented with abrupt onset of right hemiparesis. He was previously diagnosed with advanced adenocarcinoma of the prostate. Magnetic resonance imaging showed an extra-axial expansile lesion, located in the left perirolandic region associated with a nontraumatic subdural hematoma. Histopathology and immunohistochemistry of the lesion revealed metastatic prostate adenocarcinoma (Figure 1 and 2).
Dural metastasis from prostate adenocarcinoma is a rare condition, responsible for $1 \%$ of all cases ${ }^{1}$. Pathogenetic mechanisms include hematogenous spread and surgical seeding ${ }^{2}$. A dural metastasis usually presents as a solitary mass with avid contrast enhancement. Bone erosion and nontraumatic subdural hematoma may rarely be seen ${ }^{3}$.
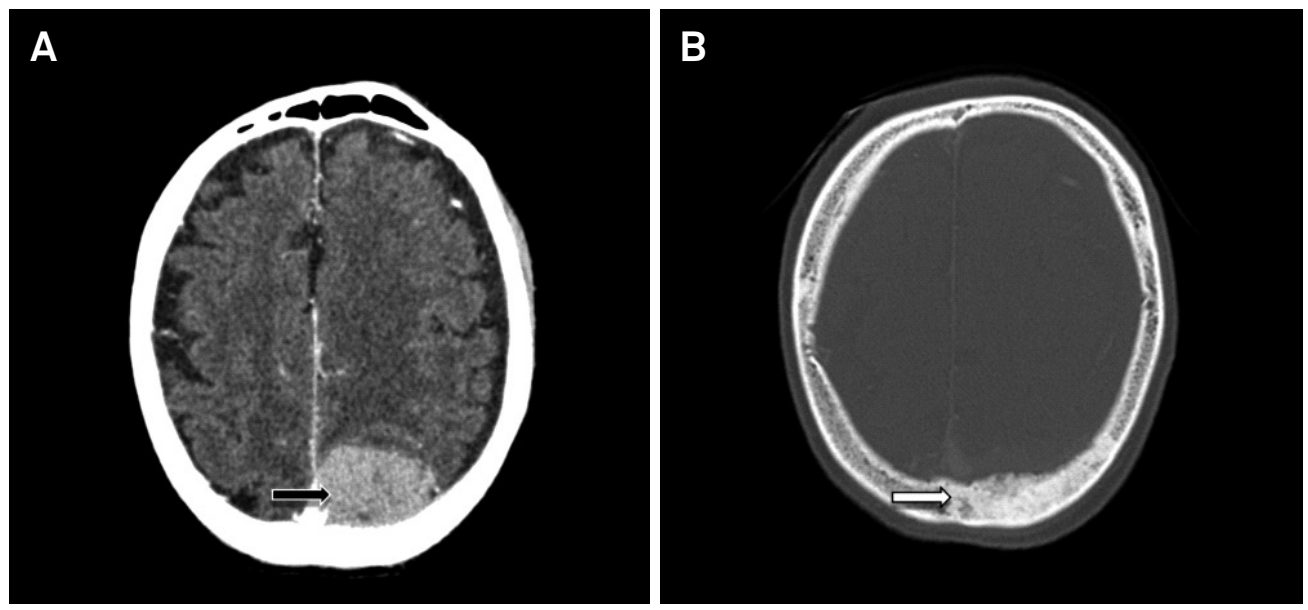

Figure 1. Computed tomography shows an oval extra-axial lesion (black arrow) on the left parietal convexity, with intense and homogeneous enhancement in the post-contrast phase (a). Note the sclerotic bone associated (white arrow), suggesting osseous infiltration (b).

'Complexo Hospital de Clínicas do Paraná, Curitiba PR, Brasil.

Marianna Cioni (ID https://orcid.org/0000-0001-5907-1477; Mateos Bogoni (iD) https://orcid.org/0000-0002-3892-3455; Carolina Chomiski (iD https://orcid.org/0000-0002-2163-5588; Laura Maschke ID https://orcid.org/0000-0003-0169-4246; Marco Aurélio Soato Ratti (iD) https://orcid.org/0000-0002-1134-084X; Bernardo Corrêa de Almeida Teixeira (iD) https://orcid.org/0000-0003-4769-6562 Correspondence: Marianna Cioni; Rua Amintas de Barros, 240 - Centro; 80060-205 Curitiba PR, Brasil; E-mail: mariannacioni@gmail.com Conflict of interest: There is no conflict of interest to declare.

Received 28 November 2018; Received in final form 05 February 2019; Accepted 20 March 2019. 

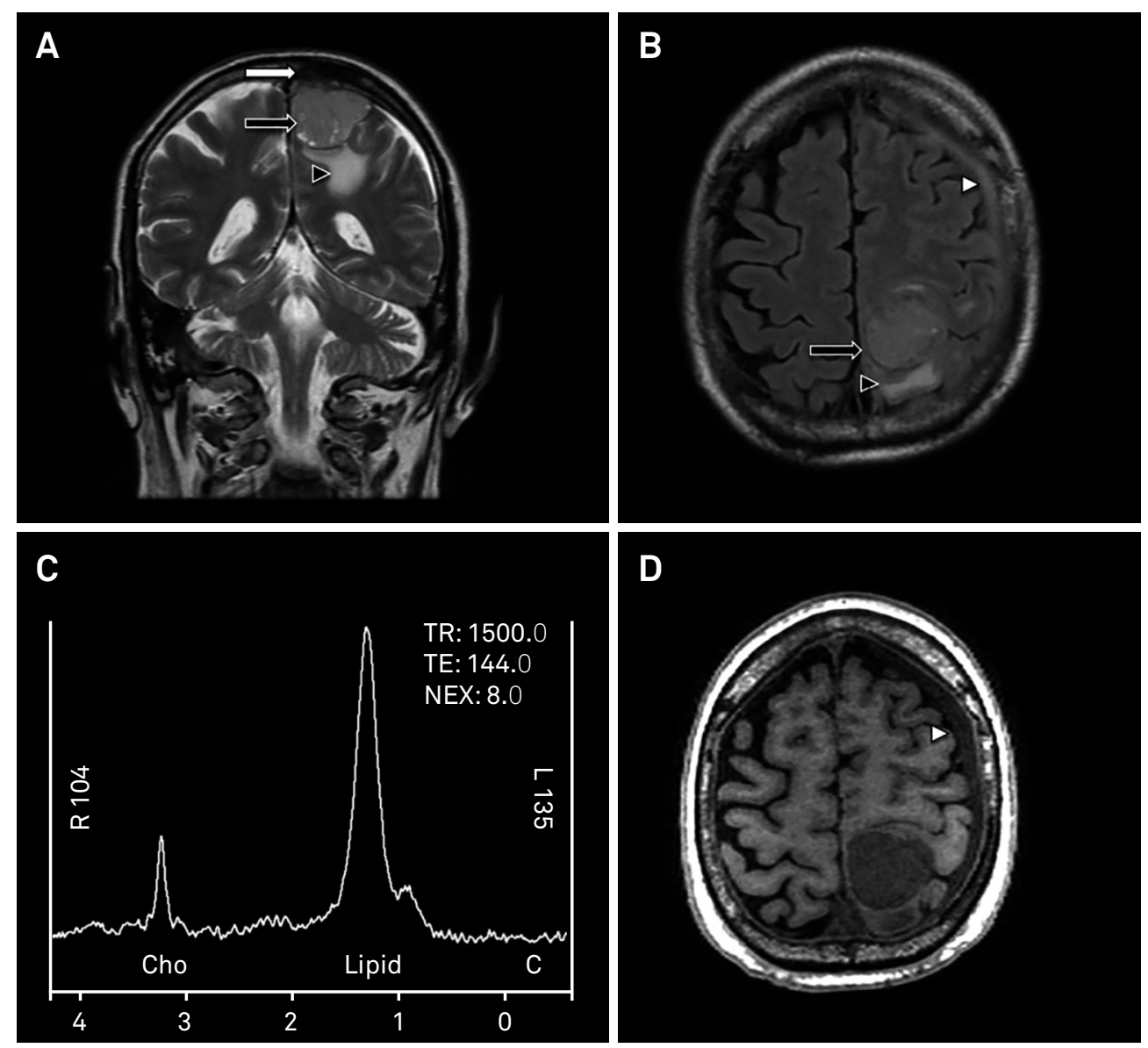

Figure 2. Magnetic resonance shows left perirolandic extra-axial expansile lesion (black arrow), hyperintense in T2-weighted images, associated with vasogenic edema (black arrowhead) and with sclerotic adjacent bone (white arrow) (a, b). Single-voxel proton spectroscopy showed absence of NAA and creatine peaks with increased choline (Cho) and large lipid/lactate peaks, suggesting metastatic origin (c). Also note left subudural hematoma (white arrowhead) (b, d).

\section{References}

1. Guedes BDVS, Rocha AJ, Gama HPP, Silva CJ. Dural metastases from prostate carcinoma: A systematic review of the literature apropos of six patients. Eur J Radiol. 2011;80(2):236-40. https://doi.org/10.1016/j.ejrad.2010.06.007

2. Lath CO, Khanna PC, Gadewar S, Patkar DP. Intracranial metastasis from prostatic adenocarcinoma simulating a meningioma. Australas Radiol. 2005;49(6):497-500. https://doi.org/10.1111/j.1440-1673.2005.01473.x

3. Smith $A B$, Rushing EJ. From the radiologic pathology archives: mass lesions of the dura: beyond meningioma: radiologic-pathologic correlation. RadioGraphics. 2014 Mar-Apr;34(2):295-312. https://doi.org/10.1148/rg.342130075 